ÉGYPTE monde arabe

\section{Égypte/Monde arabe}

27-28 | 1996

Les langues en Égypte

\title{
Réflexions sur les débuts de l'écriture dialectale en Égypte
}

\section{Madiha Doss}

\section{(2) OpenEdition \\ 1 Journals}

Édition électronique

URL : https://journals.openedition.org/ema/1928

DOI : 10.4000/ema.1928

ISSN : 2090-7273

\section{Éditeur}

CEDEJ - Centre d'études et de documentation économiques juridiques et sociales

\section{Édition imprimée}

Date de publication : 31 décembre 1996

Pagination : 119-146

ISSN : 1110-5097

\section{Référence électronique}

Madiha Doss, «Réflexions sur les débuts de l'écriture dialectale en Égypte », Égypte/Monde arabe [En ligne], 27-28 | 1996, mis en ligne le 08 juillet 2008, consulté le 07 juillet 2022. URL : http:// journals.openedition.org/ema/1928 ; DOI : https://doi.org/10.4000/ema.1928

Ce document a été généré automatiquement le 7 juillet 2022.

Tous droits réservés 


\title{
Réflexions sur les débuts de l'écriture dialectale en Égypte
}

\author{
Madiha Doss
}

1 À quelle période fait-on remonter le ou les premiers textes composés en dialecte ? La réponse ne saurait dispenser d'une réflexion sur les termes. Qu'entend-on, en effet, par «composition dialectale»? Si ces termes évoquent une production dialectale contemporaine aux manifestations multiples - poésie dialectale comme celle de 'Abd alRahmân al-'Abnûdi, de Fu'âd Negm ou de Salâh Jahîn, légendes des caricatures publiées dans la presse ou encore, textes de plus en plus nombreux des affiches publicitaires-, il faut rappeler que cette forme de composition est relativement récente dans l'histoire de l'écriture arabe : pour retrouver ses origines, il n'est pas nécessaire de remonter très loin.

2 En revanche, si l'on assimile, à la notion de composition dialectale, d'autres types d'écriture mêlant divers registres (dialectal, classique), on remonte alors beaucoup plus haut dans l'histoire de l'écrit. En effet, quelle que soit l'époque à laquelle on se réfère, il semble que l'écriture arabe présente, à différents degrés, cette variété linguistique :

- l'on trouve mêlés, dans des textes à caractère principalement classique, des éléments de vocabulaire, de morphologie ou de construction rappelant la langue parlée de différentes époques ;

- certains textes écrits en dialectal sont rarement dénués de traits appartenant à la langue écrite, littéraire.

Dans cette étude, il sera essentiellement question du premier cas. Je me propose, en travaillant sur des textes dont certains me sont familiers, de mener une réflexion susceptible d'apporter des éléments de réponse à la question ici abordée : les débuts de l'écriture en une langue proche du parler. Les textes en annexe illustreront les différentes étapes de cette réflexion. Comme on pourra le constater, ces textes datent de différentes époques. Ce choix, qui pourrait gêner la lecture, m'a semblé inévitable, certains traits ou comportements linguistiques pouvant aussi bien apparaître dans des textes contemporains que dans des écrits datant de plusieurs siècles. La quantité et la variété sont ici, à mon sens, facteur d'enrichissement. 


\section{Textes classiques incluant des traits néo-arabes}

Pour ce qui est du premier cas évoqué plus haut - celui où, dans un texte classique, apparaissent des traits proches du dialecte (ou " néo-arabes»), certains auteurs font remonter le phénomène à l'époque- et au fait lui-même -de la conquête arabe. C'est par ce facteur qu'Ibn Khaldûn explique "la corruption de la langue de Quraysh " ${ }^{1}$. En entrant en contact avec les populations conquises, les locuteurs venus d'Arabie ont développé une nouvelle forme de parler. Joshua Blau ${ }^{2}$, spécialiste de la littérature postclassique (ou « moyen arabe »), explique de la même manière le développement du néoarabe. Pour lui en effet, c'est en se propageant hors de ses frontières que l'arabe classique a subi l'évolution qu'on lui connaît, d'une langue des plus concises où le message est exprimé en peu dé termes (grâce à la vocalisation ou i'râb), à une langue où il se traduit par des mots isolés. Pour d'autres auteurs - c'est notamment le cas de Frederico C. Corriente -, l'évolution de l'arabe d'une langue avec l'ràb à une.langue sans i'râb - avec tous les autres changements que cela a entraînés - remonte avant la conquête : ces terminaisons vocaliques auraient déjà été redondantes à une période antérieure.

5 Un autre auteur, Johann Fuck, dans un ouvrage fameux sur l'histoire de ta langue et du style arabe au Xe siècle ${ }^{3}$, fait observer qu'à la période ayant suivi la chute de l'empire abbasside, « des fautes contre l'esprit de la 'arabiyya classique se rencontrent chez tous les écrivains du haut Moyen-Âge car la forme interne de leur langue était déjà du moyen arabe.»

6 En ce qui concerne l'Égypte, on sait que jusqu'au Xe siècle, c'est le bilinguisme qui y prévaut: Muqaddassi, géographe arabe de la deuxième moitié du Xe siècle, mentionne, dans sa relation de voyage, qu'on y pratique l'arabe même si le copte est encore en usage. Cependant, c'est surtout pour ta rédaction des documents officiels que l'on a recours à l'arabe, et cela depuis le vllle siècle. Par ailleurs, toujours selon Muqaddassi, la pratique de cette langue s'avère rakîka wa rakhwa («faible et négligente », du moins sur le plan grammatical), les habitants étant peu enclins à se cultiver et les fonctionnaires (kataba) étant recrutés parmi les chrétiens ${ }^{4}$.

\section{Le moyen arabe}

7 L'expression "moyen arabe », mentionnée plus haut, mérite que l'on s'y arrête un instant quoiqu'elle ait fait l'objet de maints travaux. Par ce terme à ample acception, on évoque tout texte dont la vocation est d'être écrit en classique mais où apparaissent des traits de la langue parlée ou, du moins, des aspects du classique sous-normé. Ainsi décrit-on comme moyen arabe un texte où apparaissent des traits néo-arabes ${ }^{5}$. Ces traits ont pour dénominateur commun de représenter un état de langue analytique, par opposition à la variété classique qui représente un état de langue synthétique ${ }^{6}$. Pour reprendre la définition de Blau, dans le type de langue dit "synthétique ", plusieurs concepts sont exprimés par un seul mot; alors que dans le type "analytique ", chaque mot possède une fonction indépendante. En arabe classique, le mot accompagné d'une désinence vocalique (terminaison) possède à lui seul une valeur sémantique et grammaticale. 
8 La perte des cas et désinences figure parmi les traits les plus caractéristiques de l'état de langue analytique. Comme on peut l'observer à travers les papyrus remontant aux premiers siècles après la conquête, la langue de ces écrits est exempte des terminaisons indiquant la fonction ou la distinction modale (voir par exemple texte 1 en annexe). Avec la disparition des terminaisons indiquant le cas, l'ordre des mots dans la phrase tend à devenir fixe (désormais, le sujet précède généralement le verbe). De même, l'emploi du pronom autonome (anâ) avant le verbe se généralise, alors qu'en classique, seule la forme verbale accompagnée des Suffixes pronominaux permettaient d'indiquerla personne du sujet. En raison de ce même facteur, le duel ne marque plus l'accord des verbes, des pronoms ou des adjectifs et se limite aux substantifs. Tels sont certains des traits distinguant la langue classique du néo-arabe.

9 Toute la tradition linguistique des lahn al-'amma, qui constitue un recensement des écarts par rapport à la norme grammaticale classique, indique un état de langue néoarabe.

10 II importe, dans notre tentative de définir le moyen arabe, de ne pas réduire cette variété linguistique à une somme d'«erreurs" ou d'écarts par rapport à l'écrit littéraire, pas plus qu'il ne faudrait d'ailleurs le réduire à des dialectalismes de l'époque. Le moyen arabe, c'est aussi un état de langue ,écrit qui favorise des tournures stylistiques particulières à des époques et à des lieux donnés, et dont certaines représentent des écarts par rapport à la norme littéraire, d'autres des dialectalismes, d'autres encore des développements dus à l'écrit. En voici quelques exemples :

- si l'adverbe de lieu qoddam, « devant », apparaît - bien que rarement -en classique ${ }^{7}$, son emploi fréquent est un trait typique du registre néo-arabe (moyen arabe et dialectal) ;

- la locution bi-suhbat, " avec, en compagnie de ", très fréquente dans les textes en moyen arabe d'Égypte, n'existe pas dans le parler d'aujourd'hui ;

- la construction de discours rapportés (reproduisant le discours d'autrui), dans laquelle se succèdent sans transition discours indirect et discours direct, est un trait de moyen arabe qui n'est pas nécessairement sous-tendu par le dialecte mais peut-être par des traits d'oralité (voir par exemple dans le texte 5, en annexe).

11 Nous ne nous étendrons pas davantage sur le moyen arabe; ces quelques remarques sont simplement destinées à donner un aperçu de la qualité de cette variété linguistique complexe.

\section{La littérature moyen arabe et l'écriture dialectale}

Soulignons par ailleurs que l'ensemble de la littérature moyen arabe ne peut être considérée comme représentative de la littérature dialectale ${ }^{8}$, ceci pour plusieurs raisons.

D'abord parce que dans la littérature moyen arabe, la référence linguistique est celle du littéraire - qu'il s'agisse d'écrire une lettre personnelle, une lettre d'affaires, un contrat ou une pétition -, même si les normes de ce registre ne sont que très approximativement observées (dans l'orthographe, la morphologie ou la syntaxe).

Ensuite, l'état de langue de cette littérature ne reflète sans doute que très approximativement la situation linguistique vivante, que l'on n'a aucun moyen de retrouver dans sa totalité.Pour toute personne s'étant penchée sur des textes de moyen arabe, il parait évident que cette littérature ne reflète pas le parler de l'époque. On 
sait,par exemple, que les textes moyen arabes contiennent des éléments delalangue littéraire qui ne faisaient plus partie de l'usage quotidien. Dans une chronique ottomane dont il sera question plus loin, il n'existe qu'une faible trace dela forme du pronom relatif elli (deux occurrences), alors que dans un texte à vocation clairement dialectale de la même période, cette forme, qui est celle du parler, est la plus fréquente, aux dépens de alladhi ${ }^{9}$. Cet emploi n'est pas seulement le résultat d'un phénomène d'hypercorrection ${ }^{10}$, mais plutôt d'une exigence particulière du passage à l'écrit, selon laquelle on emploie des formes littéraires à côté de formes parlées ${ }^{11}$. Il s'agit peut-être enfin d'un style d'écriture où se mêlent des traits dialectaux et des traits classiques. Ainsi, on trouvera la série des démonstratifs hadha/hadhihi utilisée àla place de da/dide la variété parlée, et ceci aussi bien dans un écrit moyen arabe remontant au MoyenÂge, que dans un texte du XIXe siècle ou même encore dans un poème dialectal écrit de nos jours (voir les exemples donnés sous le texte 7, en annexe). De même, c'est la particule lam, de l'arabe classique, qui sera employée pour la négation, plutôt que les diverses formes de négation de l'arabe parlé (mish, ma... sh, etc.).

Autrement dit, les écrits moyen arabes représentent davantage un mélange (plus ou moins codifié) de registres qu'une forme d'écriture dialectale. Effectuer un relevé des traits constitutifs de ces textes serait sans doute une des meilleures manières d'entreprendre une étude historique de l'écriture arabe.

\section{Écriture populaire et écriture dialectale}

16 Selon, une tradition académique reconnue, la variété d'écrits où se manifeste un mélange de registres mais qui reste à dominante classique est donc nommée «moyen arabe ». Je propose que l'on désigne également ce type .de textes par les termes d'écriture populaire.

17 L'avantage que cette notion ajoute à celle de moyen arabe - laquelle, reconnaissons-le, est irremplaçable -, c'est qu'elle évoque, en plus du registre ou de la variété linguistique, le groupe social et culturel des auteurs/écrivants ainsi que fa nature de leurs écrits - groupe social dont je ne saurais actuellement donner une définition satisfaisante. Un élément pourtant susceptible de caractériser ce groupe est celui de son rapport à l'écriture.

18 L'on pourrait définir certaines variétés de textes moyens arabes comme la production écrite de personnes dont le métier n'est pas l'écriture mais qui s'en servent pour les besoins de leur vie pratique (comme l'auteur du texte 1 en annexe).

19 C'est, dans d'autres cas, la production écrite de personnes qui, bien qu'ayant reçu une instruction formelle, écrivent en recourant - peut-être par choix délibéré - à un support linguistique dans lequel une part plus ou moins grande est faite aux traits du parler.

20 Au premier cas correspondraient par exemple les lettres adressées à l'Imam al-Shafî'i (voir le texte 6 en annexe) et publiées par le sociologue Sayyid 'Uways ${ }^{12}$, ou encore les lettres de la correspondance des Banu 'Abd al-Mu'min ${ }^{13}$, ainsi que lés chroniques de l'époque ottomane dont je traite plus loin. Le texte recueilli par Sayyid 'Uways est un votif rédigé par une personne sachant à peine écrire, comme le laisse soupçonner son orthographe (les mots ne sont pas séparés les uns des autres). 
21 A l'autre bout du continuum, nous trouvons les textes d'Ibn Iyyas et de Jabarti, qui écrivent dans une variété de néo-arabe où l'on retrouve de très nombreux traits caractéristiques du moyen arabe.

Entre ces deux cas, il existait - et il existe encore aujourd'hui - nombre de personnes qui se servent de l'écriture, en font même leur métier, mais dont le code de l'écrit, sans nécessairement faire place aux traits du dialecte, se rapproche de la variété orale. C'est le cas, de l'écrivain public de 'Ataba ${ }^{14}$, que j'avais présenté dans un article précédent: son écriture ne comporte pas de ponctuation et n'utilise que des wa comme termes de liaison ou d'argumentation. Cette situation est celle d'un grand nombre de fonctionnaires égyptiens, qui ont l'écriture pour métier mais qui sont plus proches des « locuteurs inexpérimentés » que de "l'éminence grise », du dominé que du dominant du moins en ce qui concerne le niveau de langue que représente leurs écrits.

En désignant ces différents types d'écriture, il importe de distinguer l'écrit personnel de celui destiné à un plus grand public. De même, il faudrait distinguer le manuscrit de l'imprimé $^{15}$. Mais encore une fois, il ne faut pas confondre écriture dialectale et écriture populaire : pour les raisons exposées plus haut, l'écriture populaire ne représente pas le parler réel d'une époque.

\section{Comment déterminer les débuts d'une écriture dialectale?}

24 Ce n'est qu'au XIXe siècle, comme on le verra plus loin, qu'apparaît le premier texte connu faisant délibérément une place importante au dialecte. Il s'agit, comme on le sait, du texte de Shirbîni, Hazz al-Quhûf. Avant cela, seuls des traits dialectaux épars apparaissent dans l'écriture. Avec quel degré de conscience? On ne saurait le dire: On peut seulement constater l'augmentation de cette tendance à employer le dialecte. $\mathrm{Si}$, dans l'écriture populaire, le mélange de registres se réduisait à des écarts par rapport à la norme classique, le degré de permissivité augmente graduellement, permettant à des structures très marquées du dialecte d'apparaître dans l'écrit à côté des structures du littéraire. Ainsi, dans un texte de nature historiographique datant du XIIIe ou XIVe siècle (voir texte 2 en annexe), apparaît la particule préverbale b-, marque dialectale par excellence, à côté des formes du littéral standard (verbe à l'inaccompli avec la terminaison un), du encore, dans ces mêmes textes ainsi que dans des poèmes de l'époque mamelouke, apparaît la forme dialectale du démonstratif $d a / d i$ à côté de la particule pré-verbale laqad, typique du registre littéral.

L'aspect dialectal ne se manifeste pas seulement dans des formes ponctuelles telles que celles qui viennent d'être citées, mais aussi dans la présence sous-jacente de traits ou de structures dialectales que l'on devine à travers les constructions littéraires ${ }^{16}$.Les exemples sont nombreux (voir en annexe,laremarque 5 du texte 1) où l'on soupçonne, sousle littéraire informel ou sous-normé, une construction dialectale.Le dialectal sousjacent au registre littéraire et perçant sous ses formes, n'est-ce pas ce qu'illustre parfaitement la tentative de trouver une langue tierce, ou la lûgha wosta, dans Al-Safqa ${ }^{17}$ ?

26 On notera finalement que les traits ou les passages en dialectal ne se limitent plus aux seuls textes poétiques; c'est dans des textes en prose que les dialectalismes apparaissent. Cette évolution mériterait qu'on s'y arrête, puisqu'on peut y voir une 
avancée de la langue parlée dans l'écrit. Tant que les traits dialectaux apparaissent dans ta poésie, cela fait partie d'une tradition littéraire acceptée et ancienne. Introduire le dialecte dans les écrits en prose, c'est lui faire une place plus importante puisqu'à ce moment, il s'agit d'élargir ses fonctions. Cette restriction du dialecte au domaine littéraire, et plus particulièrement poétique, peut s'observer encore de nos jours où l'on considère comme une transgression le fait de composer en dialectal des textes de fonctions diverses (romans, essais, critique littéraire).

On peut donc faire commencer l'histoire de l'écriture en dialectal au moment, où apparaît l'intention d'écrire dans fa langue que l'on emploie en partant. Alors que l'écriture populaire est, du moins à l'origine, non intentionnelleet remonte, comme j'ai essayé de l'expliquer plus haut, aux débuts de l'arabisation. Quant à l'initiative d'utiliser une écriture qui soitla représentation plus ou moins fidèle du parler, c'est une entreprise « moderne » et qui s'est développée graduellement.

\section{Thèmes concernant la vie ordinaire et emploi du dialecte}

28 Si je propose d'employer le terme d'intentionnalité pour distinguer, même de façon éphémère, ce qui relève à proprement parler de l'écriture dialectale et ce qui n'en relève pas, un autre élément semble s'imposer : les thèmes abordés à traversle dialecte. Il semble en effet y avoir une corrélation entre le fait de traiter de la vie quotidienne ou de thèmes frivoles ou humoristiques et l'emploi de la réserve dialectale. Ce phénomène peut s'observer à des périodes très anciennes et encore de nos jours.

29 C'est ainsi qu'Ibn al-Hajjâj (fin du IXe siècle), un des poètes de l'adab al-kudya - les "poètes gueux " ${ }^{18}$-, écrit «en contradiction consciente » avec la poésie classique lorsqu'il choisit de traiter de la vie quotidienne. «Au contenu banal correspond la forme banale: le vocabulaire de ces poésies en grande partie lascives évite toutes les expressions choisies et précieuses du style élevé, pour autant qu'il ne les parodie pas comme tous les procédés de style de l'ancienne poésie. Au lieu de cela, il utilise avec prédilection la langue de tous les jours, allant jusqu'au jargon des mendiants et des filous de Bagdad. " Ainsi l'on constate une plus grande permissivité dans le choix du lexique de la poésie de cette mouvance, qui contient des termes empruntés à la langue persane - ballur, dauraq- ou tirés de la langue courante de Bagdad - sitti (madame), rasamal (capital), shawasha (embrouiller). Même permissivité en ce qui concerne des traits néo-arabes tels que l'omission du hamza, l'emploi de l'indicatif plutôt que du subjonctif après hatta, etc.

Al Jâhiz ne recommande-t-il pas, dans Al-Bayân wa-f-Tabyîn ${ }^{19}$, que les "histoires drôles " nawâdir et tarâ'if qui mettent en scène des 'awam, "gens du peuple ", soient transcrites dans leur forme originale? «Gare à toi d'utiliser l'i'râb, :car cela nuirait à l'appréciation de l'anecdote ».

31 Cette même corrélation entre l'emploi linguistique dialectal etles thèmes relatifs au quotidien peut s'observer dans les écrits d'Ibn Sudûn, poète burlesque né au Caire en 810/1464 et dont de nombreux poèmes ont pour sujet les mets et les fruits.

Dans des pièces de zajal, genre poétique plus proche de la langue parlée que le muwashshah, sont également rapportés des faits divers, comme l'illustre cette pièce touchante transmise par Ibn Iyyas sur la mort de l'éléphant Marzûq. L'animal, qui avait 
été offert par Tamerlink à AI-Malik al-Nasîr, se noya dans le Khalîg ;l'événement, survenu en 804/1458, semble avoir beaucoup frappé les esprits de l'époque (voir texte 3 en annexe).

Ce rapport entre l'emploi du dialecte et les thèmes dela vie quotidienne, du populaire au grivois en passant par la vie des couches marginales, s'observe encore dans la littérature contemporaine. Husni Yûsuf, rédacteur du journal Lisân al-Sha'b (La voix du peuple) dans les années vingt et trente, a écrit en dialectal une série de romans inspirés par la vie de ces couches sociales: Mudhakkarat futuwwa (Mémoires d'un futuwwa), Mudhakkarat nashshal (Mémoires d'un pickpocket) ${ }^{20}$.

C'est, me semble-t-il, à une période plus récente que le registre dialectal a servi de moyen d'expression dans destextes à visée progressiste ou contestataire. Paroles de révolutionnaires, d'ouvriers et de paysans ont été versées dans le parler réel. Qantara lladhi kafar ${ }^{21}$, de Mustafa Musharrafa, qui traite de la vie des gens ordinaires durant la période ayant précédé la révolution de 1919, en est un exemple. Une partie importante de la poésie dialectale des années soixante en est un autre :je pense en particulier à la très belle œuvre de 'Abd al-Rahmân al-'Abnûdi, Ahmad lsma'în'22.

II semble également exister un certain rapport entre le dialecte et la femme, du moins en ce qui concerne l'instruction et la culture de cette dernière. Je pense ici aux écrits de 'Abd Allah Nadîm ${ }^{23}$, surtout dans Majalla al-Ustâdh, hebdomadaire créé et animé par Nadîm entre deux périodes d'exil. De très nombreux passages du journal sont rédigés en dialectal, en particulier dans les rubriques adressées aux femmes. Dans cet écrit à visée réformatrice, c'est en effet à elles que sont réservés les passages écrits en baladi (dialectal populaire) : ainsi pourront-ils être lus à celles qui ne maîtrisent pas la lecture. Sans oublier les deux romans de Bayram al-Tûnsi sur les tribulations de "Sayyid et sa femme à Paris " puis "Sayyid et sa femme en Égypte " ${ }^{24}$, textes réformateurs et comiques écrits en dialectal.

\section{La période mamelouke}

C'est cependant, semble-t-il, durant ta période mamelouke que le choix délibéré d'introduire le dialecte dans la littérature se répand, pour devenir un phénomène important. Il reste aux historiens à éclaircir la raison pour laquelle cette période est déterminante. On peut cependant avancer certaines hypothèses comme le fait Ahmad Sadîq al-Gammal, l'auteur d'un ouvrage ${ }^{25}$ sur la littérature dialectale à l'époque mamelouke. Selon lui, la littérature dialectale se serait développée lorsque les Grands parmi les Mamelouks, sultans et maîtres de maisons, qui ne maîtrisaient pas suffisamment l'arabe classique, se mirent à soutenir une production littéraire proche du parler. Toujours selon la même source, de très nombreuses écoles auraient vu le jour durant le règne des Mamelouks, fondées par ceux-ci dans le but de combattre la tradition religieuse chiite de leurs prédécesseurs fatimides. Ces nombreuses écoles sont susceptibles d'avoir créé un public détecteurs/auteurs, personnes capables de-lire et d'écrire mais qui restaient plus familières du dialecte, ou dont l'écriture était plus directement imprégnée de constructions dialectales ${ }^{26}$.

37 Le mouvement de prise du pouvoir par les Mamelouks ayant précédé leur règne proprement dit, l'insertion de ces hommes venus d'ailleurs dans ta vie linguistique et culturelle du pays pourrait remonter assez loin. La tradition consistant à recruter des Mamelouks pour servir l'État et, surtout, pour le défendre, remonte à l'époque des 
Abbassides. Les dynasties successives qui ont gouverné l'Égypte pendant tout le Moyenâge, des successeurs d'Ibn Tulûn aux Mamelouks eux-mêmes, se plieront toutes à cette tradition. Par conséquent, l'abondance d'œuvres en dialectal durant la période mamelouke n'exclut pas que ce type de composition ait pu exister plus tôt sans que l'on en ait conservé de traces importantes. Il faut par exemple mentionner un bref écrit d'Ibn Mamati, Al-fashûsh fi hukm Qaraqûsh ${ }^{27}$, qui remonte au XIIe siècle; II est difficile de classer cet écrit, car il ne représente pas à proprement parler un exemple d'écriture dialectale ni un texte moyen arabe. L'emploi du dialecte semble y être intentionnel, la nature du texte plaisante et légère. Un fait reste certain: les écrits comprenant un nombre croissant d'éléments dialectaux augmente nettement vers les XIIIe et XIVe siècles. Au début timides, dans le muwashshah, les structures s'éloignant dela norme deviennent de plus en plus dominantes dansle zajal, comme cela apparaît dans les spécimens rapportés par Ibn lyyas et qui remontent aux XIVe et XVe siècles.

\section{La période ottomane}

C'est à cette époque que l'historienne Nelly Hanna ${ }^{28}$ fait remonter l'intérêt accru pour l'écriture dialectale et les écrits populaires. Selon elle, la nature décentralisée du gouvernement, local ottoman était susceptible de stimuler la production de ces derniers. Il n'existe plus un centre unique de rayonnement de la culture, puisque l'Égypte n'est plus un centre autonome mais est devenue un satellite du sultanat d'Istanbul. Prendre la plume n'est plus le monopole de l'élite, l'écriture n'est plus le produit des seules classes dirigeantes. Le nombre de kuttâb (trois cents, selon une source dela Description de l'Égypte), la présence de nombreuses bibliothèques et l'abondance des ouvrages de vulgarisation attesteraient du fait qu'une partie de la population était capable de lire et même d'écrire sans pour autant: posséder une culture ou une instruction supérieures. N. Hanna cite Lane qui avance, dans Manners and Customs, que fort nombreux étaient les gens sachant lire et écrire, non seulement au Caire mais dans d'autres grandes villes et même dans les villages les plus étendus. Nombre de gens recevaient une instruction de base; il se serait donc constitué une classe de "lettrés » capables de s'exprimer dans une langue proche de la langue parlée (en dialectal ou en arabe populaire, ou moyen arabe); Ce qui est intéressant, c'est que ces écrits sont maintenant constitués de textes en prose et non plus seulement de poèmes, comme c'était le cas à l'époque mamelouke. Dans l'état actuel de nos connaissances, on ne rencontre pas avant le XVIIe siècle de longs textes en prose rédigés en dialectal.

En revanche, la période ottomane et plus particulièrement le XVIIe siècle offrent un vaste échantillon d'œuvres composées en dialectal ou en arabe populaire.

Je n'en citerai que deux : la première est partiellement rédigée en une forme avouée de dialectal ; la seconde est une série d'ouvrages historiographiques connus sous le titre général de chroniques de Damurdashi.

Quant à Hazz al-Quhûf fi sharh Qasîd Abî Shadûf, mentionné plus haut, c'est un des titres les plus fréquemment cités lorsqu'il s'agit de littérature dialectale ancienne. L'auteur, Yûsuf al-Shirbîni, originaire de Shirbîn (près de Mansûra), a reçu une formation à alAzhar. Hazz al-Quhûf, œuvre de compilation, traite de la vie des paysans, de leurs habitudes culinaires et vestimentaires et de leurs mœurs sexuelles dans une tradition de littérature burlesque. Hazz al-Quhûf reflète l'opposition campagne/ville, simple/ 
sophistiqué, grossier/raffiné, inférieur/supérieur : car l'avantageest toujours du coté de la ville, c'est là que se pratique la langue arabe plutôt que le "baragouinage,» des habitants des kufûr. C'est là que l'on trouve une cuisine princière contrastant avec la nourriture paysanne, l'une et l'autre décrites encore une fois avec une grande part d'exagération. Le paysan qui visite la ville a l'occasion d'épier son maître mamelouk pendant que celui-ci courtise sa femme. Il les observe dans leurs ébats amoureux pour, ensuite, mettre en pratique ce qu'il a vu. Mais, rentré chez lui, plutôt que de lancer une rose à sa femme comme il a vu son maître le faire, il lui fêlera le crâne avec une galette de bouse de vache...

Hazz al-Quhûf contient par ailleurs un long poème que Shirbîni prétend avoir recueilli auprès d'un poète local et qu'il se propose de commenter dans ce qui est une parodie du genre. Il s'agit d'un poème de zajal, en dialectal bien entendu. Le commentaire, en prose, comprend de très longs passages également en dialectal. Ce texte est considéré comme le plus important écrit dans ce registre. Comme l'explique H. Davies ${ }^{29}$ dans son étude sur la langue de cette œuvre, Shirbîni est un homme instruit, un 'âlim et un adîb ayant une vaste connaissance des sciences religieuses et littéraires; il emploie le dialectal pour amuser; Le texte est d'ailleurs conçu comme une farce.

Comme le note Nelly Hanna, des dialectalismes apparaissent également dans la littérature historiographique.

Revenons à cet égard aux chroniques de Damurdashi mentionnées plus haut : il s'agit d'un ensemble de textes écrits par différents auteurs mais qui traitent néanmoins d'un thème commun et se consacrent tous à la relation des événements advenus durant la même période (la seconde moitié du XVIIe siècle). Ecrites dans le but de divertir la caste des militaires, ces chroniques sont rédigées dans l'esprit des siyar, gestes dans lesquelles les personnages sont dotés de traits souvent, exagérés et figés. Les événements prennent parfois des allures d'épopée. Je n'entrerai pas dans le détail puisque ces chroniques, ont fait l'objet d'une étude approfondie ${ }^{30}$; je les mentionne dans la mesure où ils représentent, à mon avis, un des rares ensembles d'écrits portant si clairement des traces d'oralité. Un des textes appartenant à cette série a été écrit par Mustafa Ibrâhîm al-Qinâli qui, dans une des versions de la chronique, porte le surnom d'al-Maddah. Comme beaucoup d'autres écrits, Waqâyi' Misr al-Qâhira est un texte d'arabe populaire ou de moyen arabe comportant des traits des deux registres; mais, plus que d'autres, ce texte et les chroniques sœurs ${ }^{31}$ sont des œuvres où l'on retrouve les traces de la « vive voix » de l'écrivant, auteur ou scribe (voir texte 4 en annexe).

Dégager l'oralité dans la trace écrite arabe nous conduit également à sortir de l'étroite opposition entre dialecte et littéraire. Car que faire d'un texte dialectal portant toutes les traces de l'écriture ? Je pense en particulier au fameux Mudhakkarât tâlib bi'tha ${ }^{32}$, de Louis 'Awad. Comment le classer? Comme un texte dialectal, nécessairement. Et d'un autre côté, comment classer un texte écrit en moyen arabe, c'est-à-dire partiellement en littéraire et qui porte toutes les traces d'oralité ? Comme du moyen arabe, de l'arabe populaire; mais il me semble que le trait excessivement souligné d'oralité ne devrait pas être négligé, au risque de manquer un aspect essentiel de la langue du texte et aussi de la culture de l'époque.

Pour en revenir à nos chroniques, elles peuvent, comme je l'ai expliqué ailleurs, avoir été l'objet d'une transmission orale, les traces d'oralité retrouvées dans le texte étant alors les reliques de ce passage de l'oral à l'écrit. 

linguistique, telles que l'écrit dans l'oral et l'oral dans l'écrit, notions qui enrichiront certainement les grilles de classement des textes et mêmes de certaines .analyses dialectologiques.

Dans un ouvrage intitulé Beginnings Intention and Method ${ }^{33}$, Edward Saïd commente une citation de Giambattista Vico qu'il met d'ailleurs en épigraphe de l'ouvrage: «That beginnings are first and important but not always evident, that beginning is basically an activity which ultimately implies return and repetition rather than linear accomplishment, that beginning and beginning-again are historical whereas origins are divine, that a beginning not only creates but is its own method because it has intention. In short, beginning is making or producing difference; but - and there is the great fascination in the subject - difference which is the result or combining the already familiar with the fertile novelty of human work in language."

Cette citation me paraît très inspirante pour reposer la question soulevée par cet article. Plutôt que de chercher un point de départ seul et unique marquant le début ou l'origine de l'écriture dialectale, il serait peut-être plus prudent de rechercher les débuts multiples et variés de ce qui finit un jour par devenir une composition dialectale proprement dite. Une composition qui devient la reprise et la consécration écrites du parler, composition qui reprend le souffle de la "vive voix ». Plutôt qu'un début, on trouvera des essais différents et nombreux s'étalant sur une très longue période - allant jusqu'aux premiers textes connus - et faisant une part croissante et souvent différente aux traits dialectaux.

\section{ANNEXES}

Les textes qui suivent sont destinés à illustrer l'analyse proposée dans la présente étude. Ils figurent dans un ordre chronologique et non dans celui des références qui se trouvent dans mon texte. Les traductions sont les miennes ${ }^{34}$, excepté pour la première qui est celle des éditeurs, Anawati et Jomier.

J'ai accompagné les textes de brefs commentaires linguistiques, ce qui devrait permettre au lecteur d'observer, à partir d'extraits relativement longs plutôt que de brèves citations, quelquefois réductrices, la langue de ces écrits. Le premier texte est celui qui comporte le plus grand nombre de remarques, puisqu'il se veut un complément de l'article rédigé par Sophia Björnesjö dans ce numéro.

\section{Texte 1 : Un papyrus arabe chrétien (RR. PP. Anawati et Jomier).}

Extrait de Mélanges islamologiques II, Ifao, Le Caire, 1954 (La traduction qui suit le texte est celle des deux auteurs de l'article). 


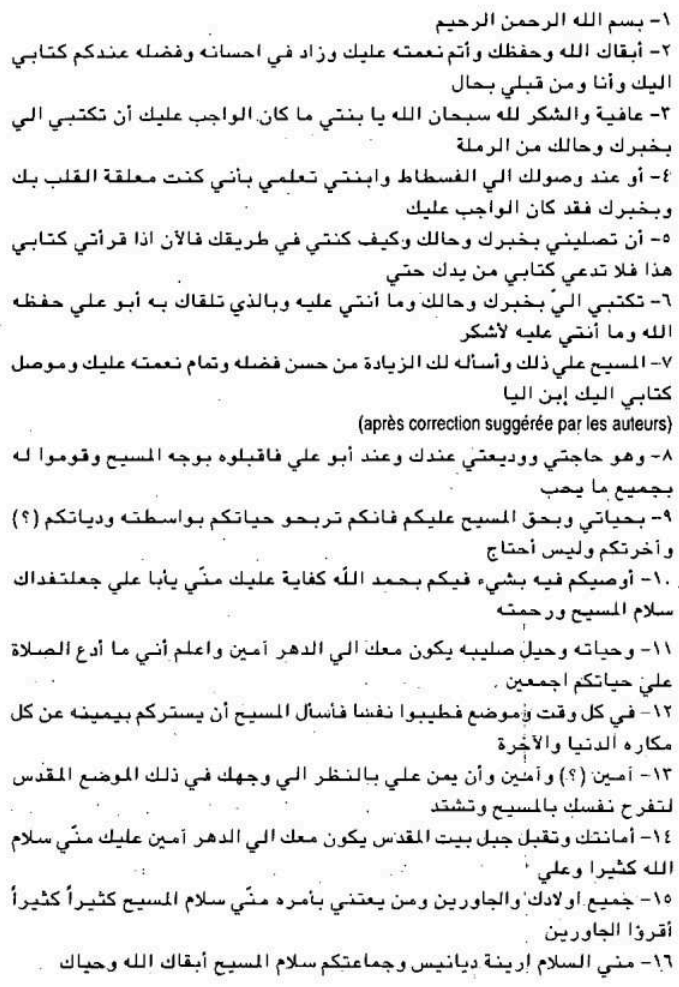

\section{Traduction :}

[1] Au nom de Dieu, le Clément, te Miséricordieux. [2] Que Dieu te conserve et te protège, qu'il te comble de sa grâce, qu' II augmente pour vous sa bienfaisance et ses laveurs. Voici ma lettre qui t'est destinée [à toi, ma fille]. Moi-même et ceux qui m'entourent, nous sommes en bonne santé, [3] Dieu merci. Par Dieu, ma fille tu aurais dû m'envoyer de tes nouvelles et [m'informer] de ta santé, soit d'al-Ramleh, [4] soit à ton arrivée à Fostat; et tu devrais savoir, ma fille, que mon cœur était suspendu à toi et à tes nouvelles. Ainsi eût-ce été un devoir pour toi [de] me faire parvenir de tes nouvelles, (de m'informer] de ton état, [de me dire] comment tu t'es trouvée en roule. Maintenant, lorsque tu auras ma lettre que voici. Ne la laisse pas quitter tes mains avant [6] de m'avoir écrit de tes nouvelles et [de m'avoir dit] ton état et comment tu te trouves, afin que j'en garde grâces [7] au Christ et que je Lui demande un accroissement de Ses laveurs si belles et le parachèvement de Ses bienfaits à ton égard. A Ibn Eliya, qui t'apporte ma lettre [8], Je me suis attachée ; il est mon dépôt auprès de toi et auprès d'Abû 'Ali, Recevez-le, par le visage du Christ, et traitez-le comme il se doit [9], par ma vie et par le droit que le Christ a sur vous. En sorte que vous gagnerez votre vie par Son intermédiaire, le prix du sang dont vous avez été rachetés [?] et votre vie de l'au-delà. Je n'ai nullement besoin [10] de vous le recommander : ce que vous avez en vous, Dieu soit loué, y suffit. Je t'envoie, ô Abu 'Ali, puisse-je être la rançon, le salut du Christ. Que sa miséricorde [11] et sa vie et la force de sa Croix soient toujours avec toi. Amen. Sache que je ne cesse de prier pour vous tous [12] en tous temps et en tous lieux, pour le bien de vos âmes, et je demande au Christ que, - par Sa droite, - Il vous protège des calamités de ce mondé et de l'autre. [13]-Amen, Amen. Et qu'il me donne de voir ton visage dans ce lieu saint pour que ton âme se réjouisse dans le Christ,-que ta confiance devienne plus forte et que les grâces d'accueil de la Montagne de Jérusalem soient toujours avec 
toi. Amen. Qu'à ma prière, la paix de Dieu soit en abondance sur toi et sur [15] tes enfants et les voisins et ceux qui ont le souci de Ses commandements; qu'à ma prière vienne la paix du Christ en surabondance. Transmettez aux voisins [16] de ma part, les salutations : à Irène, à Denis et à toute la communauté : salutations de paix du Christ. Que Dieu te garde et $\mathrm{t}^{\prime}$ accorde la vie.

\section{Commentaires.}

1) Dans leur commentaire à cette publication, Anawati et Jomier estiment que la lettre, non datée, doit remonter au IXe siècle, d'après l'écriture et les formules employées : les formules d'ouverture sont les mêmes que celles que l'on trouve dans les lettres publiées par Y. Raghîb dans la correspondance des Banu 'Abd al-Mu'min, comme par exemple XIX, p. 49 :

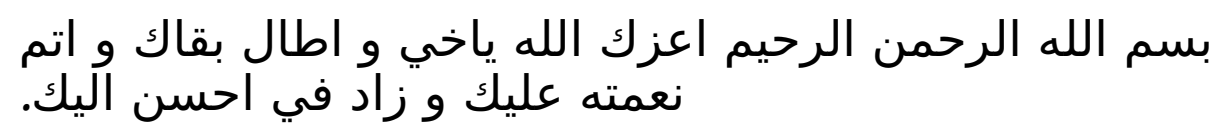

Comme le font également remarquer les auteurs, la basmala qui apparaît ici est une formule dont se servent les chrétiens comme les musulmans à cette période.

2) L'expéditrice de la lettre est une chrétienne, comme le montrent clairement les حيل صليبه : mentions du Christ et le rappel d'autres éléments religieux dans le texte etc. Hormis ces formules religieuses, la langue du texte s'identifie à toute autre lettre du même genre qui aurait été écrite par un .musulman. Cette absence de particularité linguistique identifiant une communauté religieuse est aujourd'hui un fait bien connu, contredisant les premières analyses proposées par J. Blau qui voyait le moyen arabe comme la production écrite des non-musulmans. S. Bjômesjô, pour sa part, explique dans son article (voir dans le présent numéro) que l'arabe a été intégré assez tôt dans la culture égyptienne et est devenu, dès le IXe siècle, la norme de tout écrit

3) Un des traits caractéristiques de ce type de texte est l'emploi fréquent de la forme du masdar plutôt que de la forme conjuguée du verbe. Un exemple très commun du style épistolaire est l'expression كتلبي اليك qui est systématiquement employée pour signifier Cette expression représente une des formules introductives dans la correspondance de cette période, comme par exemple dans une des lettres de la collection des Banu 'Abd al-Mu'min :

المحبه لك مني قايمه على ما تستحقه و لمعرفتي بمحبتك و كثره مسلك عني

«Mon amitié pour toi est constante, comme tu le mérites, et parce que je connais ton amitié et la fréquence avec laquelle tu demandes de mes nouvelles. »

Ce trait peut être expliqué de diverses manières, notamment comme un effet de la simplification du système verbal. Pour une explication générale des effets de la simplification sur le système verbal, on peut consulter l'ouvrage de K.Versteegh sur la « pidginization ${ }^{35}$,

4) Comme c'est le cas encore aujourd'hui, on rencontre de nombreux exemples d'emploi des particules get ف dans l'écriture arabe. Ces éléments de ligature, également particules de coordination, ont un rôle important pour relier entre eux des énoncés séparés. 
5) Des expressions peuvent être construites à partir de traits de la langue littéraire tout en étant dialectales dans leur conception sous-jacente :

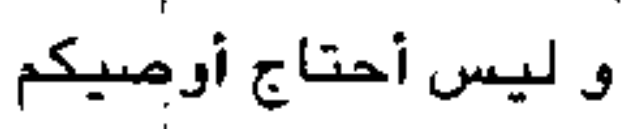

Cet énoncé, constitué strictement d'éléments du registre littéraire, recouvre en fait une construction dialectale sous-jacente avec l'outil de négation $m a$, sans doute plus fréquent dans le parler mais qui, justement pour cette raison, est écarté de l'usage écrit. C'est la forme qui est employée dans une construction qui n'obéit pas à la norme.

\section{Texte 2 : Zettersteen, Beiträge sur Geschishte des Mamlüken Sultane in des Jahren 690-741 des Higra nach arabischen Handschritten, Leiden, 1919.}

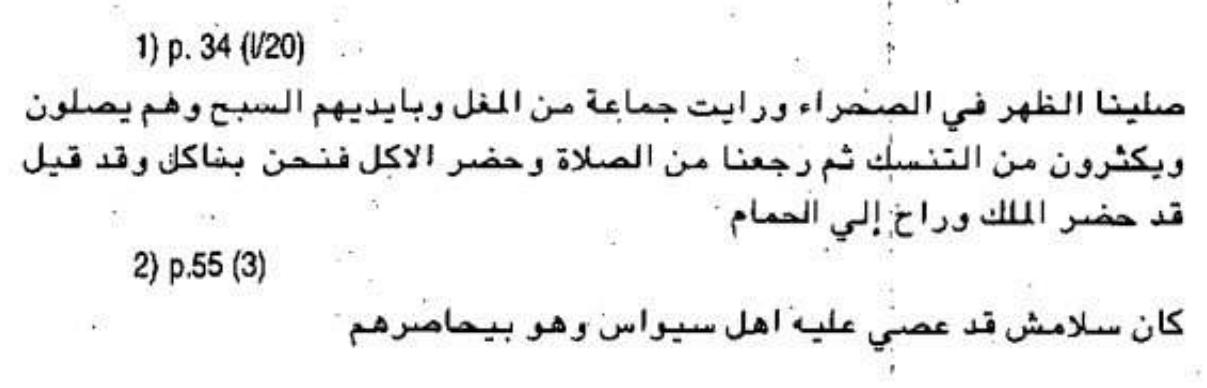

\section{Traduction}

1) «Nous avons accompli la prière de midi dans le désert, et j'ai vu un groupe de Moghols, des chapelets à la main, qui priaient et redoublaient de dévotion, Puis, revenus de la prière, le repas fut servi ; pendant que nous mangions, on annonça que le roi était arrivé et qu'il était allé au hammam. »

2) «Les habitants de Siwa résistaient à Salamesh tandis qu'il les assiégeait. »

\section{Commentaire}

On notera dans les deux énoncés l'apparition de la particule préverbale baccompagnant le verbe à l'inaccompli et exprimant une action qui se déroule en même temps qu'une autre (concomitante). Cette particule très spécifique de la variété dialectale est employée dans un contexte où apparaissent aussi des traits exclusif de la variété littéraire tel que הَّ. 


\section{Texte 3 : Extrait d'Ibn lyyas, Badâ'îl al-zuhûr, 1 ère impression, al-Amiriyya (?)/1311.}
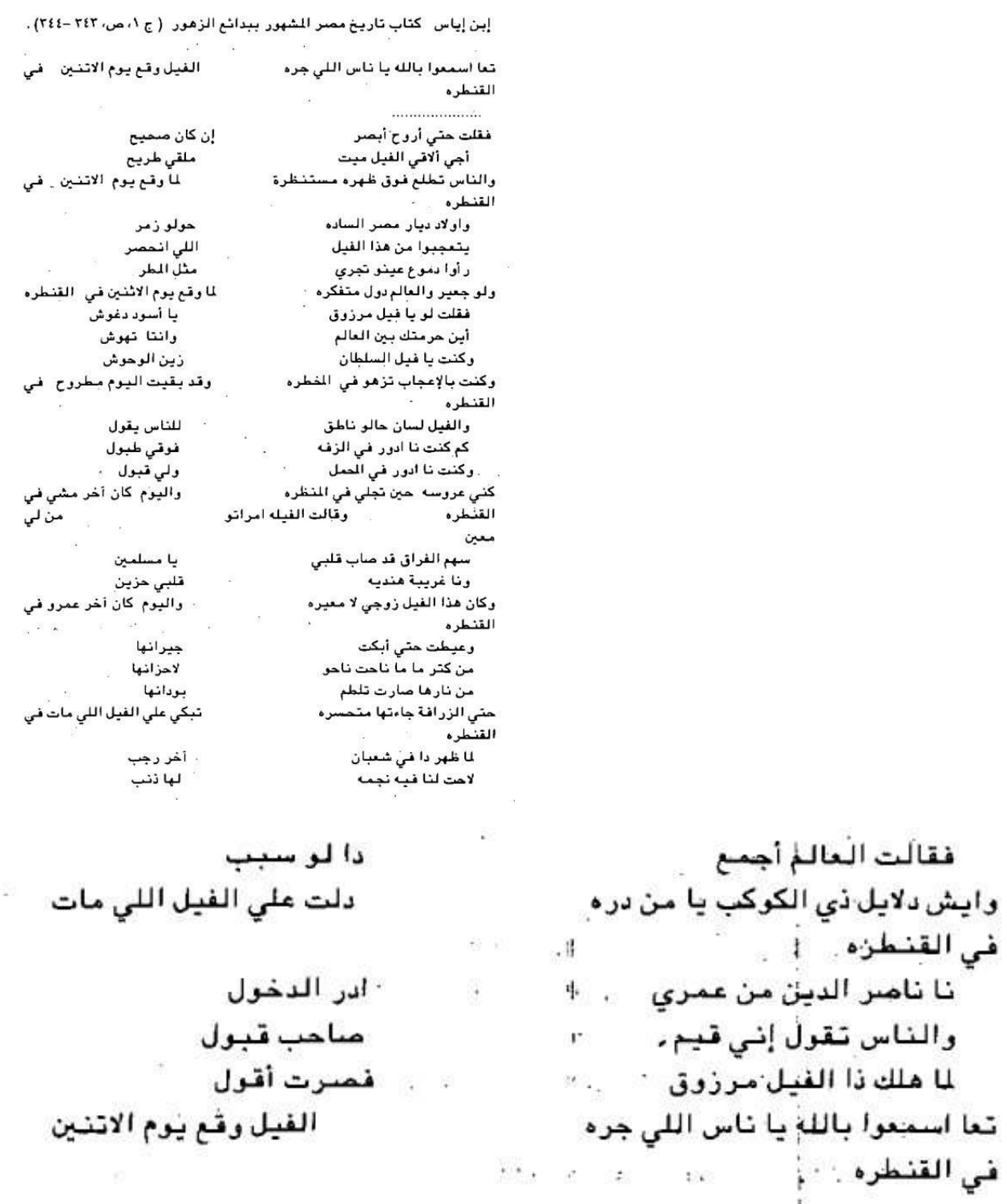

\section{Traduction}

Par Dieu, approchez, venez écouter, vous autres

Ce qui est arrivé à l'éléphant tombé lundi sur le pont

Je me dis: allons voir si c'est vrai

J'arrive et trouve l'éléphant mort, étendu à terre.

Et les gens de lui grimper sur le dos

Alertés lorsqu'il tomba lundi sur le pont 
Et les habitants vénérés du Caire, massés autour de lui

Etonnés de voir cet éléphant en détresse

Voient ses larmes couler comme la pluie

Ils pensent : «Quels barrissements il a dû pousser

Quand sur le pont il a basculé

Je lui dis : « Ô toi, éléphant Marzûq

Toi, noir comme la nuit

Qu'es-tu devenu, toi l'intouchable?

Semant autour de toi la peur

Tu étais, toi l'éléphant du sultan.

La plus belle des bêtes féroces

Tous admiraient la grâce de la démarche

Et te voilà aujourd'hui abattu sur le pont. »

L'éléphant comme éveillé dit aux gens :

«Souvenez-vous lorsque Je circulais en procession

Portant les tambours battants

J'avançais, le palanquin sur le dos, et tous m'acclamaient

Comme une mariée lorsqu'elle paraît à la fenêtre

Et voilà qu'aujourd'hui

Ce fut ma dernière promenade sur le pont. »

L'éléphante, sa compagne, dit :

«Qui me viendra en aide?

La flèche de la séparation a fendu mon cœur, ô musulmans !

Moi qui suis étrangère, venue d'Inde, mon cœur est affligé

Cet éléphant était mon compagnon, j'en suis flattée

Mais aujourd'hui, ce fut son dernier Jour sur le pont. »

Elle se mit à pleurer

Ses pleurs tirèrent des larmes aux voisins

Elle gémit tant et tant qu'ils gémirent de son chagrin

De douleur elle se lamentait, se frappant de ses oreilles

La girafe vint même à son secours, affligée,

Pleurer l'éléphant mort sur le pont.

Lorsque cela advint, durant le mois de sha'ban, fin ragab

On vit paraître une étoile filante 
Les gens pensèrent qu'il s'agissait d'un signe

Que présageait donc cette étoile filante? On se le demanda.

Un signe de la mort de l'éléphant sur le pont...

Je m'appelle Nasir al-Dîn, et toute ma vie, j'amasse de l'argent

Les gens m'apprécient, j'ai même du succès

Lorsque mourut cet éléphant Marzûq, je récitai :

«Par Dieu, approchez, venez écouter, vous autres,

Ce qui est arrivé à l'éléphant tombé lundi sur le pont!»

\section{Commentaires}

1) Cette histoire touchante, racontée sous la forme d'un poème de zajal, est rapportée par Ibn Iyyas, qui rappelle que le jour de l'accident, les marchands du bazar ont fermé leurs échoppes pour aller assister à l'événement. Dans la tradition du zajal, le dernier vers reprend le premier et le nom du poète est désigné dans la dernière strophe.

2) Sur le plan de l'orthographe, on peut observer le souci de se rapprocher de la phonétique, comme dans le premier mot du poème, " approchez », تعا, avec l'ellipse d'une partie de ce mot qui serait normalement تعالو. Même ellipse de la première syllabe dans Li, ici écrit LJ comme il apparaîtrait dans le flot de la parole : kunt'ana, Le son /a/ est écrit à l'aide de la lettresه, qui représente un alif ou un yấ' dans l'orthographe normaleoر جره يا من

Le pronom suffixe de la 3e personne est dans de nombreux cas transcrit par un واو او لو- عينو- حولو mais dans d'autres cas, on trouve l'orthographe normale : ظهره.

Ces quelques remarques ne concernent que le plan des sons et la manière de les transcrire mais loin de s'arrêter là, l'intérêt du poème est multiple et, ne serait-ce que sur le plan du son, on est frappé par l'aspect musical du poème. Le zajal était d'ailleurs un genre poétique très lié à la chanson.

3) J'ai été frappée de rencontrer la dixième forme ou dans le mot

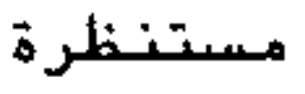

. Cette forme, plus fréquente dans le registre «populaire » que dans le registre «commun ${ }^{36}$ du dialecte, semble donc remonter à une période assez ancienne.

4) On notera finalement le mélange des variétés - trait dialectal suivi de trait littéral. Comme on le voit encore aujourd'hui, cette hétérogénéité est un des traits de la poésie

dialectale. Ainsi, on trouve le démonstratif de la série classique

quelques vers plus loin, des formes dialectales

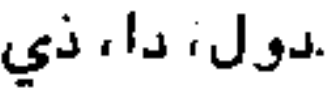

d'autres d'époques, la place du démonstratif dans ce texte est hésitante : il est placé 
tantôt avant, tantôt après le nom :

\section{.العالـم دول، ذا الـفيـل}

proposé une explication à cette variation ${ }^{37}$, selon laquelle la position préposée (avant le nom) du démonstratif serait plus marquée que l'autre, ce qui apparaît encore aujourd'hui dans un emploi figé, celui des imprécations, ou dans certaines

variation de la place du démonstratif est encore fréquente dans la poésie dialectale. Hétérogénéité de niveau également dans l'emploi de la particule de comparaison

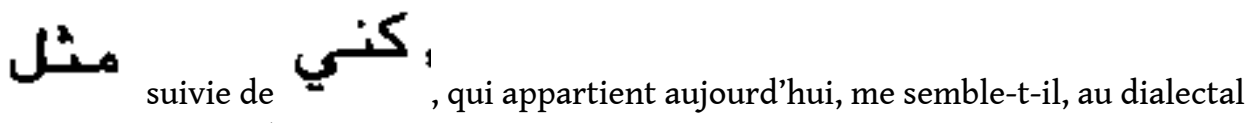
sa'idi (du sud de l'Égypte).

\section{Texte 4 : Texte manuscrit sans titre, mais généralement désigné par un énoncé figurant dans la première ligne. La copie porte la cote H.0.38 de la Bibliothèque nationale de Vienne.}

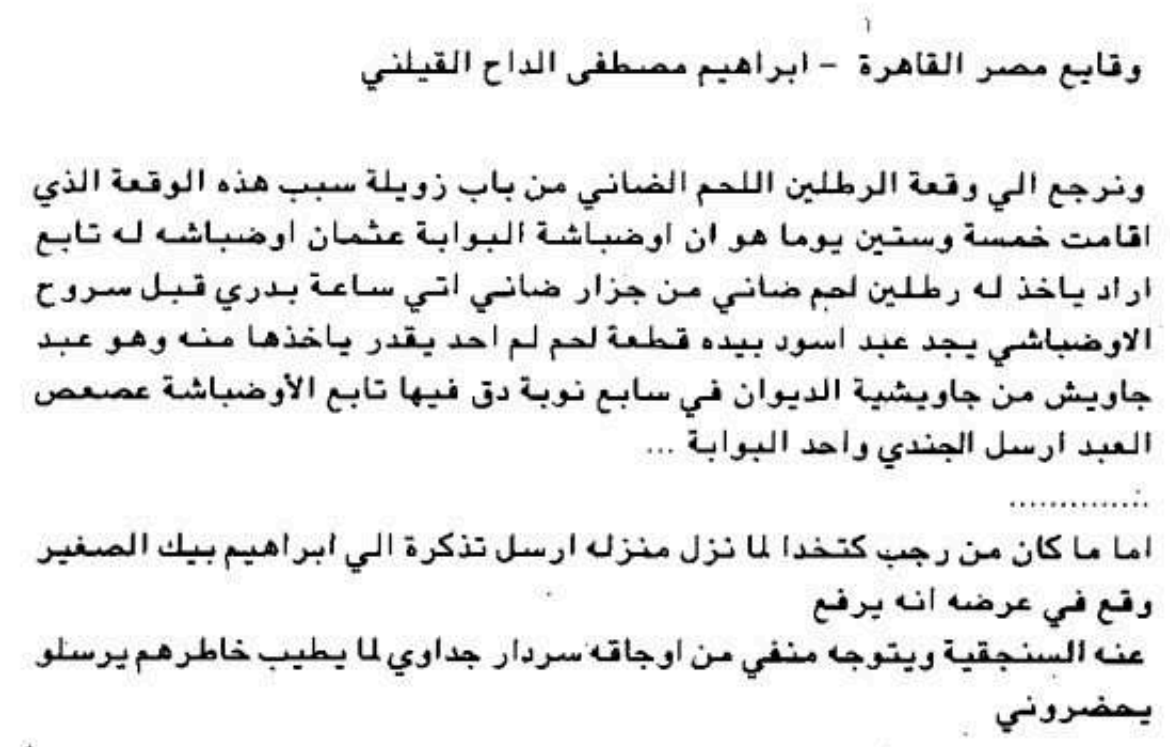

\section{Traduction}

« Revenons à l'affaire des deux ratl [près d'un quart de kilo, N.D.T.] de viande de mouton de Bâb Zuwayla. La raison de cette querelle, qui dura soixante-cinq jours, c'est qu'un odabash ${ }^{38}$ de la milice, nommé 'Uthmân Odabasha, avait un serviteur. Celui-ci voulut prendre pour son maître deux ratl de viande chez un boucher qui vendait du mouton. Il arriva une heure avant la tournée de son maître l'odabash pour trouver un esclave noir, un morceau de viande à la main. Personne n'arriva à le lui arracher. Cet esclave était celui d'un des jawish ${ }^{39} \mathrm{du}$ palais de la septième brigade de musique. Le 
serviteur saisit le morceau de viande, l'esclave lui échappa, le soldat fit envoyer quelqu'un au quartier général de la milice...

Quant à Rajab Katkhoda, arrivé chez lui, il envoya un message à Ibrâhîm bek al-Saghîr, le suppliant de lui ôter le titre de sandjaq et [de .lui permettre] de se diriger vers Jadda en tant qu'exilé de son odjaq ».

\section{Commentaires}

1) On peut observer l'emploi de la négation لم لم احد يقدر ياخدها employée contrairement à la norme, comme dans l'exemple commenté plus haut (texte 1, commentaire 3). On entrevoit, à travers la forme littéraire la construction dialectale sans doute déjà employée puisque la négation ماحدش... sh apparaît dans d'autres textes de la même période, comme Hazz al-Quhûf et d'autres versions de cette même chronique, ...

2) On notera que le pronom relatif الذي est au masculin alors que son antécédent gest féminin. C'est que la forme effectivement employée était le dialectal, comme on le sait par d'autres textes, Ici, c'est la forme de l'écrit qui s'impose.

3) L'emploi de l'inaccompli (ou présent historique) pour rapporter un événement du passé semblerait être plus ancien qu'on ne le pense. En effet, dans de nombreux passages narratifs du manuscrit, on trouve des formes telles que يجد Ce qui tendrait à prouver que cet emploi ne s'explique pas par l'influence de là prose journalistique en langues occidentales ${ }^{40}$.

\section{Texte 5}

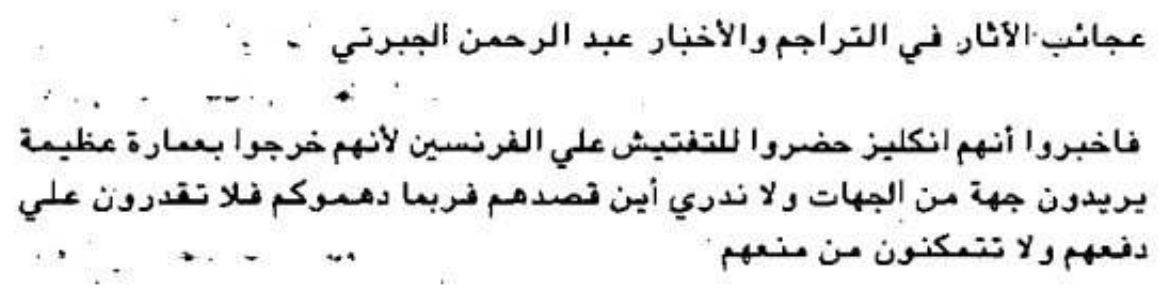

\section{Traduction}

Ils ont annoncé qu'ils étaient Anglais et qu'ils étaient à la recherche des Français. Ceuxci étaient partis sur un énorme navire vers quelque destination, nous ignorons vers où ils se dirigeaient. Peut-être vous envahiraient-ils, alors vous seriez incapables de les en empêcher et ne sauriez point les repousser.

\section{Commentaire}

On notera la forme de discours rapporté dans ce très bref passage de l'histoire de Jabarti, qui relate les événements relatifs à la campagne de Napoléon. Le passage du discours indirect au discours direct s'effectue sans transition. En effet, le début de la 
réponse des Anglais est rapporté au discours indirect, introduit par la conjonction suivie du pronom suffixe انهم, puis on passe brusquement au discours direct avec ندري. Cet exemple rappelle des usages encore observables de nos jours dans la prose journalistique, où les transformations exigées par le discours direct ne sont souvent pas maintenues, trait d'écriture sans doute très marqué par la pratique orale. La même remarque s'applique également au deuxième extrait de Qîlani (texte 4).

\section{Texte 6 : Lettre anonyme recueillie à Al-Mahalla dans la province de Gharbiyya, le 18 octobre 1956. Les explications entre parenthèses sont celles de Sayyid ‘Uways.}

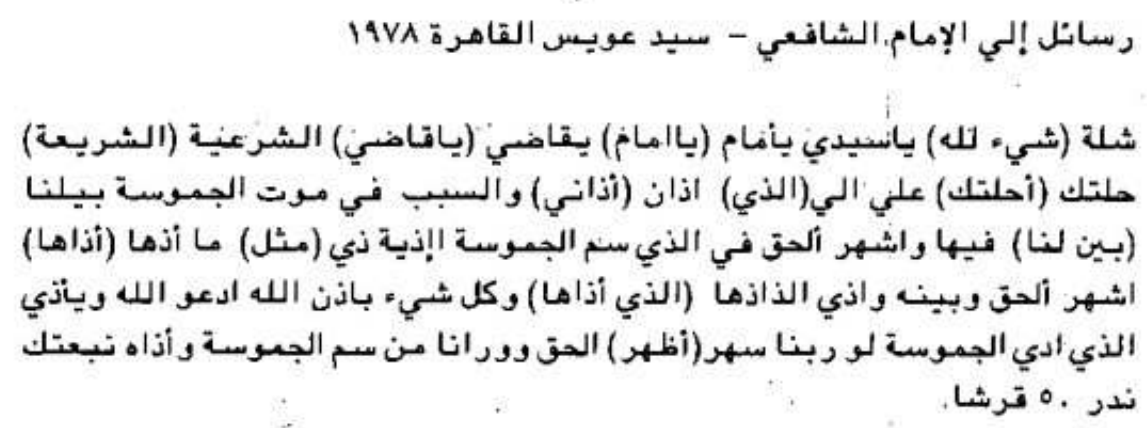

\section{Traduction}

«A la faveur de Dieu, vous, imam de la justice, je vous charge d'agir auprès de celui qui m'a fait du tort et a été la cause de la mort de la bufflesse, découvrez-le pour nous et faites justice à celui qui a empoisonné la bufflesse, faites-lui du tort comme il en a fait à notre bufflesse, faites apparaître la justice, découvrez-le et nuisez à celui qui lui a fait du tort, Tout sera fait selon la volonté de Dieu, j'invoque Dieu pour qu'il fasse du mal à celui qui a nui à la bufflesse, Si Dieu fait surgir la vérité et qu'il nous montre qui a empoisonné la bufflesse et qui lui a fait du tort, je fais vœu de vous envoyer 50 piastres.

\section{Commentaires}

1) Votif écrit par une personne maîtrisant mal l'écriture, comme le montre

lorthographe très variable le

du vocatif est tantôt lié au reste du mot comme dans

tantôt séparé du mot comme dans
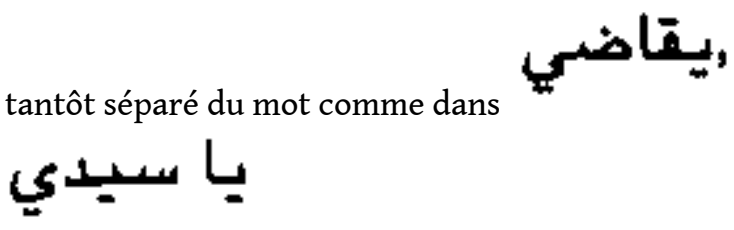

, tantôt séparé du mot comme dans 
2) On constate la même variation pour la forme du pronom relatif

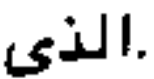

hésitation typique des périodes où le code n'est pas encore bien assimilé par les locuteurs relève plutôt ici de l'absence de maîtrise des registres linguistiques. L'auteur de ce texte est inexpérimenté dans l'emploi du code écrit.

\section{Texte 7}

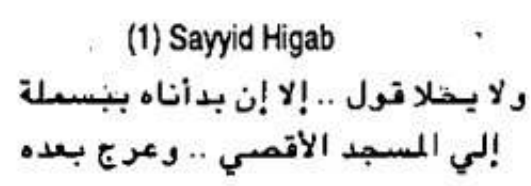

(2) 'Abd al-Rahmân al-'Abnúdi

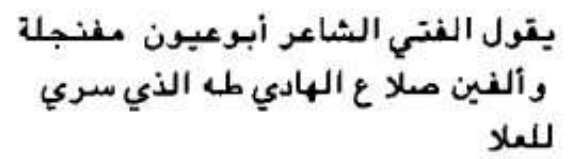

للبلا

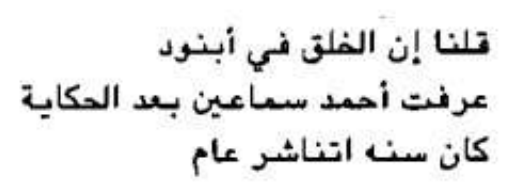

\section{Traduction}

(1) « Il dit, le jeune homme aux yeux écarquillés, et à quoi sert de parler si l'on ne commence par la basmala

Et deux mille prières sur le Prophète, qui alla à la mosquée d'al-Aqsa puis s'éleva dans les cieux. »

(2) « Nous avons dit que les gens, à Abnûd,

avaient connu Ahmad Ismâ'îl après l'histoire

Il avait douze ans. »

\section{NOTES}

1. An Arab Philosophy of History, Selection from the Prolegomena of IBN KHALDUN (1332-1406) translated and arranged by Charles Issawi, AUC Press, 1987.

2. BLAU Joshua, "The Beginnings ot the Arabic Diglossia. A Study of the Origins of Neoarabic ", Studies in Middle Arabic and its Judeo-Arabic Variety, Jérusalem, 1988, p. 8-9. Même référence, CORRIENTE.

3. FUCK Johann, 'Arabiya. Recherches sur l'histoire de la langue et du style arabe, trad. C. Denizeau, Paris, 1955, p. 144.

4. Ibid., p. 165.

5. A propos de la différence entre les deux termes de néo- et de moyen arabe : le premier a été utilisé par Blau pour indiquer aussi bien les dialectes arabes modernes que le moyen arabe, tout 
en reconnaissant, bien entendu, la différence des circonstances extralinguistiques ayant conduit à l'un et à l'autre. Le moyen arabe est utilisé par le même auteur pour parler des textes anciens.

6. BLAU Joshua. "The Importance of Middle Arabic Dialects for the Hislory of Arabic », p. 69, "The beginnings,.. », p. 2 et «Classical Arabic, Middle Arabic, Middle Arabic Literary Standard, Neo-Arabic, Judeo-Arabic and Related Terms ", Studies in Middle Arabic..., op. cit., p. 255-259.

7. BLAU Joshua, A Grammar of Christian Arabic, Louvain, 1966, p. 247, 153.

8. Une erreur .très fréquente, chez les non-spécialistes, consiste à prendre pour dialectaux des traits de moyen arabe. Par exemple, l'idée est répandue que dans les écrits de Jabarti, l'on trouve du dialectal. En fait ce sont le plus souvent, chez cet historien, des traits qui n'appartiennent pas plus au dialectal qu'au littéraire mais qui constituent les éléments du style particulier d'une époque (voir exemples dans l'annexe).

9. Les textes comparés ici sont, d'une part, celui de la chronique de Qinali (voir référence plus bas) et le célèbre Hazz al-Quhûf de Yûsuf al-Shirbîni.

10. Joshua Blau a donné une définition de ce phénomène dans plusieurs de ses écrits. Il s'agit de l'emploi de traits de la langue envisagée comme «supérieure » à la place de traits associés, dans l'esprit des locuteurs, à la langue parlée qui, elle, est dévalorisée.

11. «C'est une erreur de croire qu'un texte écrit puisse être l'exacte représentation de la parole. Contrairement à l'opinion de bien des gens, on n'écrit jamais comme l'on parle; on écrit (ou l'on cherche à écrire) comme les autres écrivent On peut ça et là retrouver des traits dialectaux, cela va sans dire, mais la distance de cet écrit au parler reste grande. » VENDRYÈS, Le langage, p. 325-326.

12. 'UWAYS Sayyid, Rasà'il ila-l-lmam al-Shafíi, Dar al-shayi' li-l-nashr, Le Caire, 1978.

13. RAGHEB Yûsuf, Marchands d'étoffes du Fayyoum au IIIe/IXe siècle d'après leurs archives (Actes et lettres). Les actes des Banu 'Abd al Mu'min, Le Caire, 1982.

14. DoSS Madiha, « Des écrivains publics à 'Ataba ». Égypte/Monde arabe n 14, 2e trimestre 1993.

15. Distinction faite en 1936 par Jean Lecerf, qui notait que c'est à l'introduction de l'imprimerie «qu'il faut faire remonter l'origine de changements notables de la situation respective des deux formes d'arabe », et qui prévoyait déjà que « la naissance et le développement d'un important commerce de librairie [pouvait) suffire à modifier les conditions de la vie intellectuelle ». LECERF Jean, «Littérature dialectale et renaissance arabe moderne », Bulletin d'études orientales, 1932, t. 2, fasc. 2, Institut français de Damas, p. 190-191.

16. AL-BASHÎR IBN SALÂMA, Nadhariyyât al-ta'tîm al-iqấ'i fi-l-fusha, Al-dâr al-tûnisiyya li-l-nashr, 1984. L'auteur de l'ouvrage propose d'analyser et d'exploiter le potentiel qu'offrent les différents registres de la langue arabe.

17. AL-HAKÎM Tawfîq, Al-Safqa, Le Caire, 1981.

18. Mentionné par Fuck, 'Arabiya, op. cit., p. 155 sq.

19. RAWHY AL-FAYSAL Samar, "Lughat al-hiwâr ti-l-adab », Al-fikr al-'arabi, al-lugha al-'arablyya wa-ishkâlat al-mu'àsara n 60, avril-juin 1990.

20. YÛSUF Hosni, Mudhakkarât futuwwa, Le Caire, 1930 ; Mudhakkarât nashshal, Le Caire, 1930.

21. MUSHARRAFA Mustafa, Qantara lladhi kafar, Kitâb adab wa naqd, Le Caire, 1991.

22. AL-'ABNÙDI 'Abd al-Rahmân, Ahmad Isma'în sirit insân, Le Caire, 1972.

23. NADIM 'Abd Allah, Majallat al-Ustâdh, Al-hay'a al-'amma li-l-kitàb, Le Caire, 1994.

24. AL-TÛNSI Bayram, Al-Sayyid wa-m'ratu fi Bàrîs et Al-Sayyid wa-m'ratu fi Masr, Al-maktaba al-'asriya, Sayda, Beyrouth, s. d.

25. SADÎQ AL-GAMMAL Ahmad, Al-adab al-'ammi fi Misr fi-'asr al-mamlûki, Al-dâr al-qawmiya li-ltibâ'a wa-l-nashr, 1966.

26. Argument avancé par Nelly Hanna concernant la période mamelouke.

27. Ibn Mamati, Al-fashûsh fi hukm Qaraqùsh, éd. 'Abd al-Latîf Hamza, Le Caire, s.d.

28. HANNA Nelly, "Culture in Ottoman Egypt », Cambridge History of Modern Egypt, vol. II, à paraître. 
29. DAVIES Humphrey Taman, Seventeenth-Century Arabic: A Profile of the Colloquial Material in Yûsuf al-Shirbîni's « Hazz at-Quhuffi Sharh Qasîd Abi Shadûf », University of California, Berkeley, PhD 1981, p. 5-7.

30. DOSS Madiha, L'arabe en Égypte. Étude évolutive d'une langue de relation, thèse de doctorat d'État, Paris III, Paris, 1991 (non publiée).

31. Al-Durra al-musana fi akhbar al-Kinâna, édition annotée par 'Abd al-Rahmân 'Abd al-Rahîm, Ifao, Le Caire, 1988.

32. 'AWAD Louis, Mudhakkarât tâlib bi'tha, Al-hay'a al-'amma li-l-kitâb, Le Caire, 1991.

33. SAÏD Edward, Beginnings, Intention and Method, Basic Books, Inc. Publishers / New York, 1975. La citation de Giambattista Vico est la suivante : «Doctrines must take their beginning from that of the matters of which they treat ", (The New Science, 1744).

34. Je remercie Menha el-Batrawi pour l'aide qu'elle m'a apportée dans la traduction du texte 3 (extrait d'lbn Iyyas, Badâ'îl al-Zuhûr).

35. VERSTEEGH Kees, Pidginization and Creolization. The Case of Arabic, Amsterdam, 1984.

36. Les termes entre guillemets ont le défaut de n'avoir pas reçu de définition claire. En l'absence d'une classification plus précise, retenons celle d'Al-Sa'îd Badawi, chez qui l'on trouverait, à la place des termes que j'emploie, ceux de

37. Doss Madiha, «The Position ot the Demonstrative da, di in Egyptian Arabic: A Diachronic Inquiry », Annales islamologiques, t. XV, Ifao, 1979.

38. Chef de chambrée.

39. Soldats de la milice (...) qui avaient l'emploi de chanter devant le sultan, dans ses marches solennelles (définition de DOZY, Supplément aux Dictionnaires arabes, Paris, 3e édition, 1967).

40. SOMECK Sasson, Genre and Language in Modern Arabic Literature, Weisbaden, 1991, p. 33.

\section{INDEX}

Mots-clés : arabe (langue), dialecte (arabe), linguistique

\section{AUTEUR}

\section{MADIHA DOSS}

Université du Caire 